\title{
Autoreporte de la calidad de vida relacionada con la salud en diabetes mellitus tipo 2
}

\author{
ALFONSO URZÚA M. ${ }^{\mathrm{a}}$, ALEJANDRA CHIRINO ${ }^{\mathrm{b}}$, \\ GERALDINE VALLADARES ${ }^{b}$
}

\section{Health related quality of life among patients with type 2 diabetes mellitus}

Background: Type 2 diabetes mellitus may affect profoundly the quality of life of patients. Aim: To assess health related quality of life among patients with Type 2 Diabetes Mellitus. Material and Methods: The Diabetes Quality of Life (DQOL) questionnaire was applied to 296 patients with diabetes mellitus aged $63 \pm 10$ years (201 women) seen in primary health care centers. Results: The concern about the future effects of diabetes was the worst evaluated domain. Women perceived a lower health related quality of life than men. There was an inverse correlation between age and satisfaction with treatment, concern about vocational, social and future effects of the disease. Conclusions: Type 2 diabetes affects health related quality of life, especially in some specific domains such as perception of the future.

(Rev Med Chile 2011; 139: 313-320).

Key words: Diabetes mellitus, type 2; Quality of life; Questionnaires.
Escuela de Psicología, Universidad Católica del

Norte, Antofagasta, Chile. apsicólogo, Magíster en Salud Pública, Doctor en Psicología Clínica y de la Salud.

bPsicóloga.

Recibido el 15 de abril de 2010, aceptado el 4 de enero de 2011.

Correspondencia a: Dr. Alfonso Urzúa M. Avenida Angamos 0610. Antofagasta. Chile. E-mail: alurzua@ucn.cl
I a diabetes mellitus (DM) es una de las enfermedades crónicas prevalentes en Chile, comunicándose prevalencias de $4,2 \%{ }^{1}$ a $5,4 \%^{2}$ en población general. El $80 \%$ de los casos de DM son clasificables en el tipo 2 (DM2) $)^{3,4}$.

La DM2 acorta la expectativa de vida, empeora el pronóstico funcional y se asocia a complicaciones potencialmente letales ${ }^{5}$. Los pacientes atraviesan períodos de tensión emocional al someterse a restricciones dietéticas y físicas, con serias repercusiones emocionales y sociales ${ }^{6}$, afectando todos los aspectos de su vida ${ }^{7}$.

El tratamiento es de por vida, pudiendo la enfermedad provocar complicaciones en los pacientes $^{8}$, afectando seriamente su calidad de vida relacionada con la salud (CVRS), entendida ésta como el nivel de bienestar derivado de la evaluación que la persona realiza de diversos dominios de su vida, considerando el impacto que en éstos tiene su estado de salud ${ }^{9}$.
Existe evidencia que la DM2 afecta negativamente la CVRS ${ }^{5,10,11}$. Diversos factores se han reportado vinculados a una mejor o peor CVRS en personas con DM2 tales como el sexo ${ }^{5-8,12}$, edad ${ }^{5,6,11}$, la presencia de una pareja ${ }^{12}$, nivel de estudios ${ }^{6-8}$, apoyo social ${ }^{13}$, índice de masa corporal ${ }^{5,14}$; complicaciones derivadas ${ }^{5-7,10}$, comorbilidades asociadas $^{14}$, tipo de tratamiento y satisfacción con éste ${ }^{8}$, control glucémico ${ }^{8,14}$ y la satisfacción con la salud ${ }^{11}$.

En Chile no se han reportado estudios sobre CVRS en pacientes con DM2 adultos desde la perspectiva del propio paciente. En este marco, el objetivo general de esta investigación es evaluar factores relacionados con el autoreporte de la CVRS en pacientes diabéticos, a la vez que conocer la percepción del adulto con DM2 sobre su CVRS, identificar aspectos que influyen en la CVRS de los pacientes diabéticos y determinar los dominios de la CVRS que son más afectados por esta enfermedad. 
Método

La investigación se realizó bajo un diseño no experimental, de tipo transversal.

\section{Participantes}

Entre los meses de mayo y septiembre, se invitó a participar en la investigación a 420 pacientes que tenían hora para acudir al control de crónicos en los consultorios de Atención Primaria de la ciudad de Antofagasta. Estos cumplían los criterios de inclusión de contar con un diagnóstico de DM2, reportado por ellos mismos y con un tiempo de evolución superior a 1 año. Esto luego fue corroborado con la ficha clínica de los que accedieron a participar. Se excluyeron personas con problemas mentales o del sistema nervioso central que dificultasen su capacidad de comprensión. Del total invitado a participar, accedieron a completar los cuestionarios 296 personas.

\section{Instrumento}

Se utilizó un instrumento compuesto de dos partes. La primera sección indagó datos socio demográficos y de salud. La segunda sección correspondió al cuestionario específico Diabetes Quality of Life (DQOL) ${ }^{17}$. El DQOL consta de 48 preguntas agrupadas en cuatro dimensiones: satisfacción con el tratamiento ( 15 reactivos), impacto del tratamiento (20 reactivos), preocupación por los efectos futuros de la diabetes (4 reactivos), preocupación por aspectos sociales y vocacionales (7 reactivos), más una pregunta sobre el estado de salud percibido. El puntaje total obtenido se transforma a un índice de 0 a 100, donde los puntajes más bajos indican que el paciente tiene una mejor percepción de CVRS en esa dimensión.

La versión en castellano de este instrumento ha demostrado adecuados índices de consistencia interna y una estructura factorial acorde a la propuesta teórica del instrumento ${ }^{15}$.

En la presente investigación, la consistencia interna (medida a través del Alpha de Cronbach) fue de 0,81 para el dominio satisfacción con el tratamiento; 0,76 para impacto del tratamiento; 0,71 para preocupación por aspectos sociales y vocacionales, 0,68 para preocupación por efectos futuros de la diabetes y 0,86 para la CV (Total de DQOL).

\section{Procedimiento}

Una vez aprobada la investigación por el Comité de Bioética de la Universidad Católica del Norte y del Departamento de Salud de la Corporación Municipal de Desarrollo Social, se realizó la coordinación necesaria con los establecimientos de Atención Primaria.

Cada cuestionario fue administrado individualmente, previa firma del consentimiento informado. El tiempo promedio de aplicación fue de 35 minutos. Posteriormente, se accedió a las fichas clínicas de los pacientes para obtener información relevante requerida para la investigación.

Los datos fueron ingresados a una base construida en SPSS.12. Una vez aplicada la sintaxis de corrección de la escala, se realizó un análisis descriptivo de las dimensiones que componen el DQOL, para luego correlacionar o analizar diferencias de medias, dependiendo de la variable a relacionar. Dada la amplitud del rango de tiempo transcurrido desde el último examen realizado, se consideró para el análisis sólo los datos de pacientes con exámenes de sus niveles de glucosa y colesterol tomados en los últimos tres meses.

\section{Resultados}

\section{Participantes}

La Tabla 1 contiene algunas características de los participantes. La muestra estuvo compuesta mayoritariamente por mujeres (201 participantes $-68 \%)$. La media de edad fue de 63,1 años (DE = 10,4 años), con un rango de edad que osciló entre los 23 y los 80 años.

El tiempo transcurrido desde el diagnóstico varió entre 1 y 59 años, con un tiempo promedio viviendo con la enfermedad de 8,7 años ( $\mathrm{DE}=8,6$ años). El peso de los participantes estuvo entre los 48 y los $140 \mathrm{~kg}$, siendo el promedio de peso de los hombres significativamente mayor que el de las mujeres $(r=0,03 ; p<0,05)$.

Del total de fichas de las que se pudo obtener información acerca del último control (101, equivalente a $34 \%)$, sólo $47(15 \%)$ tenían datos de exámenes realizados en los últimos tres meses para el colesterol y para glucosa.

\section{Calidad de vida}

La Tabla 2 contiene los promedios de las puntuaciones brutas y estandarizadas de la escala, así como los puntajes brutos reportados en el estudio español ${ }^{15}$. Al comparar los puntajes brutos obtenidos en ambas poblaciones, la media reportada por 
Tabla 1. Características de los participantes

\begin{tabular}{|c|c|c|c|c|c|c|}
\hline & \multicolumn{2}{|c|}{ Muestra total } & \multicolumn{2}{|c|}{ Hombre } & \multicolumn{2}{|c|}{ Mujer } \\
\hline & $\overline{\mathrm{x}}$ & DE & $\overline{\mathrm{x}}$ & DE & $\overline{\mathrm{x}}$ & DE \\
\hline Edad en años & 63,13 & 10,42 & 63,39 & 9,99 & 63,02 & 10,6 \\
\hline Años de estudio & 9,54 & 4,06 & $10,57^{*}$ & 3,63 & 9,06 & 4,67 \\
\hline Personas que viven con el paciente & 3,34 & 2,40 & 3,15 & 2,12 & 3,43 & 2,52 \\
\hline $\begin{array}{l}\text { Personas que apoyan al paciente en los cuidado de } \\
\text { su enfermedad }\end{array}$ & 1,92 & 1,81 & 1,87 & 1,43 & 1,94 & 1,97 \\
\hline $\begin{array}{l}\text { Personas que viven con el paciente, consideradas } \\
\text { importantes por él }\end{array}$ & 3,05 & 2,16 & 2,95 & 2,03 & 3,09 & 2,23 \\
\hline Años desde el diagnóstico de la enfermedad & 8,75 & 8,67 & 8,79 & 8,93 & 8,73 & 8,56 \\
\hline Índice de Masa Corporal & 29,87 & 5,92 & 28,01 & 3,78 & 30,78 & 6,55 \\
\hline Peso (kg) & 76,27 & 14,70 & $79,18^{*}$ & 11,52 & 74,84 & 15,87 \\
\hline Glicemia último control (0 a tres meses) (mg/dl) & 171,34 & 122,6 & 159,47 & 71,06 & 176,90 & 141,16 \\
\hline \multirow[t]{2}{*}{ Colesterolemia último control ( 0 a tres meses) $(\mathrm{mg} / \mathrm{dl})$} & 188,58 & 48,99 & 161,56 & 45,44 & 199,63 & 46,92 \\
\hline & Frec. & $\%$ & Frec. & $\%$ & Frec. & $\%$ \\
\hline $\begin{array}{l}\text { Vive con pareja } \\
\text { Vive solo }\end{array}$ & $\begin{array}{l}178 \\
118\end{array}$ & $\begin{array}{l}60 \\
40\end{array}$ & $\begin{array}{l}70 \\
25\end{array}$ & $\begin{array}{l}74^{1} \\
26\end{array}$ & $\begin{array}{r}108 \\
93\end{array}$ & $\begin{array}{l}54 \\
46^{1}\end{array}$ \\
\hline $\begin{array}{l}\text { Hipertensos } \\
\text { No Hipertensos }\end{array}$ & $\begin{array}{r}233 \\
63\end{array}$ & $\begin{array}{l}79 \\
21\end{array}$ & $\begin{array}{l}77 \\
18\end{array}$ & $\begin{array}{l}81 \\
19\end{array}$ & $\begin{array}{r}156 \\
45\end{array}$ & $\begin{array}{l}78 \\
22\end{array}$ \\
\hline $\begin{array}{l}\text { Con complicaciones } \\
\text { Sin complicaciones }\end{array}$ & $\begin{array}{l}124 \\
172\end{array}$ & $\begin{array}{l}42 \\
58\end{array}$ & $\begin{array}{l}33 \\
62\end{array}$ & $\begin{array}{l}35 \\
65\end{array}$ & $\begin{array}{r}91 \\
110\end{array}$ & $\begin{array}{l}45 \\
55\end{array}$ \\
\hline
\end{tabular}

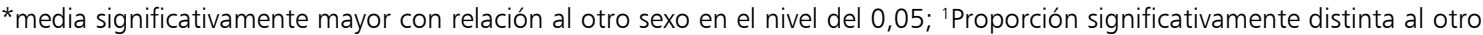
sexo $(p<0,05)$.

Tabla 2. Medidas de tendencia central CVRS general y Dominios estudios Chile-España

\begin{tabular}{|c|c|c|c|c|c|c|c|c|}
\hline \multirow[b]{2}{*}{ Dominio } & \multicolumn{2}{|c|}{$\begin{array}{c}\text { Población } \\
\text { chilena } \\
\text { (PE } 0 \text { - 100) }\end{array}$} & \multicolumn{3}{|c|}{$\begin{array}{l}\text { Población chilena } \\
\text { (PB) }\end{array}$} & \multicolumn{3}{|c|}{$\begin{array}{l}\text { Población española } \\
\text { (PB) }\end{array}$} \\
\hline & $\overline{\mathrm{x}}$ & DE & Frec & $\bar{x}$ & DE & Frec & $\bar{x}$ & DE \\
\hline Satisfacción con el tratamiento & 38,80 & 12,37 & 296 & 29,08 & 9,25 & 13 & $31,18^{*}$ & 9,37 \\
\hline Impacto del tratamiento & 38,86 & 10,17 & 296 & 38,86 & 10,17 & 101 & $44,08^{* *}$ & 10,69 \\
\hline $\begin{array}{l}\text { Preocupación por aspectos sociales } \\
\text { y vocacionales }\end{array}$ & 14,62 & 11,52 & 296 & 5,09 & 4,05 & 132 & $6,03^{*}$ & 5,28 \\
\hline $\begin{array}{l}\text { Preocupación por efectos futuros } \\
\text { de la diabetes }\end{array}$ & 41,13 & 16,58 & 296 & 8,23 & 3,32 & 134 & 8,14 & 3,91 \\
\hline Calidad de vida (total del DQOL) & 32,85 & 9,50 & 296 & 79,26 & 21,07 & 80 & $92,67 * *$ & 22,13 \\
\hline
\end{tabular}

Frec $=$ Frecuencia; *media significativamente mayor que la de la población chilena para similar dimensión en un nivel de confianza del $95 \% ;{ }^{* *}$ media significativamente mayor que la de la población chilena para similar dimensión en un nivel de confianza del $99 \%$. 
Tabla 3. Medidas de tendencia central de puntajes por dominio por sexo y estado de pareja

\begin{tabular}{|c|c|c|c|c|c|c|c|c|}
\hline & \multicolumn{4}{|c|}{ Sexo } & \multicolumn{4}{|c|}{ Estado } \\
\hline & \multicolumn{2}{|c|}{ Hombre } & \multicolumn{2}{|c|}{ Mujer } & \multicolumn{2}{|c|}{ Con pareja } & \multicolumn{2}{|c|}{ Sin pareja } \\
\hline & $\bar{x}$ & DE & $\bar{x}$ & DE & $\bar{x}$ & DE & $\overline{\mathrm{x}}$ & DE \\
\hline Satisfacción con el tratamiento & 35,82 & 11,88 & $40,20 * *$ & 12,37 & 38,12 & 12,72 & 39,80 & 11,78 \\
\hline Impacto del tratamiento & 33,91 & 8,56 & $38,25^{* *}$ & 10,58 & 36,57 & 10,05 & 37,30 & 10,37 \\
\hline $\begin{array}{l}\text { Preocupación por aspectos sociales } \\
\text { y vocacionales }\end{array}$ & 15,93 & 12,91 & 13,99 & 10,77 & 13,82 & 10,74 & 15,82 & 12,54 \\
\hline $\begin{array}{l}\text { Preocupación por efectos futuros de } \\
\text { la diabetes }\end{array}$ & 35,00 & 14,43 & $44,02 * *$ & 16,76 & 41,20 & 15,99 & 41,01 & 17,49 \\
\hline Calidad de vida (Total DQOL) & 30,16 & 8,99 & $34,12^{* *}$ & 9,50 & 32,43 & 9,30 & 33,48 & 9,81 \\
\hline
\end{tabular}

** Medias significativamente mayores que la de los varones a 99\% de confianza.

la pacientes españoles es superior en los dominios de satisfacción con el tratamiento $(t=2,188 ; \mathrm{p}<$ $0,05)$, impacto con el tratamiento $(\mathrm{t}=4,396 ; \mathrm{p}<$ $0,01)$, preocupación con aspectos sociales y vocacionales $(\mathrm{t}=2,012 ; \mathrm{p}<0,05)$ y en el puntaje total de la escala $(t=4,997 ; \mathrm{p}<0,01)$.

Considerando que un puntaje menor equivale a una mejor percepción de CVRS, el dominio peor evaluado corresponde a la preocupación por efectos futuros de la diabetes, seguido por la satisfacción y el impacto del tratamiento. El puntaje más bajo obtenido fue en la subescala preocupación por aspectos sociales y vocacionales, implicando que la CV de las personas con DM2 se ve menos afectada en esta dimensión.

\section{CVRS y variables sociodemográficas}

$\mathrm{Al}$ analizar las medias obtenidas en los diversos dominios de acuerdo al sexo (Tabla 3), se encontraron diferencias estadísticamente significativas en los dominios satisfacción con el tratamiento $(\mathrm{t}=-2,881(294), \mathrm{p}<0,01)$, impacto del tratamiento $(\mathrm{t}=-3,76(223,75), \mathrm{p}<0,01)$, preocupación por efectos futuros de la diabetes $(t=-4,516(294)$, $\mathrm{p}<0,01)$ y en la CV total $(\mathrm{t}=-3,46(193,99)$, $\mathrm{p}<0,01)$. En todas estas, las mujeres percibían una peor CVRS que los hombres. Los hombres obtuvieron un mayor puntaje en la subescala preocupación por aspectos sociales y vocacionales, sin embargo, esta diferencia no fue significativa.

$\mathrm{Al}$ correlacionar los puntajes obtenidos en los distintos dominios con la edad, a nivel de la muestra total, se encontró que existe una relación inversa entre ésta y los dominios satisfacción con el tratamiento $(\mathrm{r}=-0,129, \mathrm{p}<0,05)$, preocupación por aspectos sociales y vocacionales $(r=-0,179, p<0,01)$, preocupación por efectos futuros de la diabetes $(\mathrm{r}=-0,125, \mathrm{p}<0,05)$ y CV total $(\mathrm{r}=-0,171, \mathrm{p}<$ 0,01 ), esto es, a mayor edad, mejor CV percibida. $\mathrm{Al}$ analizar esta relación por sexo, sólo se encuentra relaciones estadísticamente significativas en las mujeres, en donde la edad correlaciona negativamente con la dimensión preocupación por aspectos sociales y vocacionales $(\mathrm{r}=-0,203, \mathrm{p}<0,01)$ $y$ en $C V$ total $(r=-0,162, p<0,05)$, esto quiere decir que las mujeres de mayor edad percibían una mayor $\mathrm{CV}$ en estas dimensiones.

A nivel de la muestra total no se encontraron diferencias significativas entre las medias obtenidas por las personas con pareja y sin pareja (Tabla 3). Al estratificar el análisis por sexo, sí se encontraron diferencias entre las medias reportadas por hombres con y sin pareja en el dominio Satisfacción con el tratamiento, en donde la media de los que tienen pareja fue significativamente menor (mayor CVRS) que la de aquellos que no la tienen $(\mathrm{t}=-2,316(93) ; \mathrm{p}<0,05)$. Entre las mujeres no se encontraron diferencias.

Se encontró relación entre el número de personas que viven con el paciente y el dominio impacto del tratamiento $(\mathrm{r}=0,128, \mathrm{p}<0,05)$, donde a mayor cantidad de personas viviendo con el paciente, menor $\mathrm{CV}$ percibida en ese dominio. $\mathrm{Al}$ estratificar por sexo, se encontró que en las mujeres correlacionan significativamente con el número de personas la dimensión impacto del tratamiento 
Tabla 4. Medidas de tendencia central de puntajes por comorbilidad de hipertensión y presencia de complicaciones

\begin{tabular}{|c|c|c|c|c|c|c|c|c|}
\hline & \multicolumn{4}{|c|}{ Comorbilidad hipertensión } & \multicolumn{4}{|c|}{ Presencia de complicaciones } \\
\hline & \multicolumn{2}{|c|}{ Sí } & \multicolumn{2}{|c|}{ No } & \multicolumn{2}{|c|}{ Sí } & \multicolumn{2}{|c|}{ No } \\
\hline & $\bar{x}$ & DE & $\bar{x}$ & DE & $\bar{x}$ & DE & $\bar{x}$ & DE \\
\hline Satisfacción con el tratamiento & 38,3 & 11,6 & 40,7 & 14,8 & 42,4 & 13,1 & 36,2 & 11,2 \\
\hline Impacto del tratamiento & 37,2 & 10,3 & 35,7 & 9,9 & 40,7 & 11,1 & 34,1 & 8,5 \\
\hline $\begin{array}{l}\text { Preocupación por aspectos sociales } \\
\text { y vocacionales }\end{array}$ & 13,8 & 9,7 & 17,7 & 16,3 & 17,1 & 13,1 & 12,8 & 9,9 \\
\hline $\begin{array}{l}\text { Preocupación por efectos futuros } \\
\text { de la diabetes }\end{array}$ & 40,9 & 16,8 & 42,1 & 16,0 & 47,7 & 17,7 & 36,4 & 13,8 \\
\hline Calidad de vida (Total DQOL) & 32,5 & 9,1 & 34,0 & 11,0 & 37,0 & 10,4 & 29,9 & 7,6 \\
\hline
\end{tabular}

$(\mathrm{r}=0,171, \mathrm{p}<0,05)$ y la CV total $(\mathrm{r}=0,143, \mathrm{p}<$ $0,05)$. Esto quiere decir que las mujeres percibían una peor $\mathrm{CV}$ en lo relacionado a impacto del tratamiento y $\mathrm{CV}$ total, mientras el número de personas con quienes vive aumenta. En los hombres no se encontraron relaciones estadísticamente significativas.

A nivel de la muestra total, se encontró una correlación negativa entre el número de personas que apoyan al paciente en el cuidado de su enfermedad y la dimensión satisfacción con el tratamiento $(r=-0,134, p<0,05)$. Esto sugiere que a mayor cantidad de personas que vivan y lo apoyen en el cuidado de su diabetes, estos refieren una mayor satisfacción con el tratamiento. No se encontraron correlaciones significativas al evaluar por sexo. No se encontró relaciones significativas tanto en la muestra total como al estratificar por sexo al analizar la relación de los diversos dominios con el número de personas que viven con el paciente y son importantes para él o con los años de estudio de los pacientes.

\section{CVRS y variables biomédicas}

Se encontró correlación entre los años desde el diagnóstico y la dimensión impacto del tratamiento $(\mathrm{r}=0,147, \mathrm{p}<0,05)$, implicando que a mayor cantidad de años, menor CVRS percibida en ese dominio. Al estratificar este análisis por sexo, esta correlación se mantuvo sólo en las mujeres $(\mathrm{r}=$ $0,143, \mathrm{p}<0,05)$.

No se encontraron diferencias significativas en la CVRS entre las personas que presentan hiper- tensión arterial y las que no tenían el diagnóstico (Tabla 4).

Los participantes que presentaron complicaciones percibían una peor $\mathrm{CV}$ en los dominios satisfacción con el tratamiento $(\mathrm{t}=4,389$ (294); $\mathrm{p}<0,01)$, impacto del tratamiento $(\mathrm{t}=5,48$ $(220,36) ; \mathrm{p}<0,01)$, preocupación por aspectos sociales y vocacionales $(t=3,10(218,15) ; p<0,01)$, preocupación por efectos futuros de la diabetes $(\mathrm{t}$ $=5,88(221,44) ; \mathrm{p}<0,01 ;)$ y en la CV total $(\mathrm{t}=$ $6,47(213,28) ; \mathrm{p}<0,01)$ que los que no reportan complicaciones (Tabla 4).

Aun cuando se observa una menor CVRS en las diversas dimensiones en aquellos participantes con obesidad clase III, no se encontraron correlaciones significativas entre el peso de los participantes y su IMC con los puntajes obtenidos en las distintas dimensiones evaluadas (Tabla 5). Tampoco se ha encontrado diferencias en las medias de los dominios evaluados de la CV al evaluar las diferencias entre el grupo con valores de glicemia controlado y aquellos que superaban el valor esperado de 130 $\mathrm{mg} / \mathrm{dl}$.

\section{Discusión}

Se analizó la relación entre variables sociodemográficas y biomédicas con el autoreporte de CVRS en pacientes con DM2.

El dominio más afectado correspondió a la preocupación por efectos futuros de la diabetes, posiblemente debido a las potenciales complica- 
Tabla 5. Medidas de tendencia central dimensiones de CV por IMC

\begin{tabular}{|c|c|c|c|c|c|c|c|c|c|c|}
\hline & \multicolumn{2}{|c|}{$\begin{array}{c}\text { Saludable } \\
\text { IMC } 18,5-24,9 \\
(41)\end{array}$} & \multicolumn{2}{|c|}{$\begin{array}{c}\text { Sobrepeso } \\
\text { IMC 25-29,9 } \\
\text { (89) }\end{array}$} & \multicolumn{2}{|c|}{$\begin{array}{c}\text { Obesidad } \\
\text { clase I } \\
\text { IMC 30-34,9 } \\
\text { (57) }\end{array}$} & \multicolumn{2}{|c|}{$\begin{array}{c}\text { Obesidad } \\
\text { clase II } \\
\text { IMC 35-39,9 } \\
\text { (29) }\end{array}$} & \multicolumn{2}{|c|}{$\begin{array}{l}\text { Obesidad } \\
\text { clase III } \\
\text { IMC > } 40 \\
(17)\end{array}$} \\
\hline & $\overline{\mathrm{x}}$ & DE & $\overline{\mathrm{x}}$ & DE & $\bar{x}$ & DE & $\overline{\mathrm{x}}$ & DE & $\bar{x}$ & DE \\
\hline Satisfacción con el tratamiento & 38,1 & 11,4 & 37,3 & 12,8 & 40,6 & 14,1 & 37,4 & 12,7 & 42,6 & 9,6 \\
\hline Impacto del tratamiento & 36,6 & 11,3 & 36,8 & 10,9 & 37,1 & 9,5 & 39,6 & 9,0 & 40,2 & 10,3 \\
\hline $\begin{array}{l}\text { Preocupación por aspectos } \\
\text { sociales y vocacionales }\end{array}$ & 14,6 & 14,0 & 14,9 & 13,1 & 13,7 & 10,7 & 13,6 & 9,7 & 14,9 & 9,4 \\
\hline $\begin{array}{l}\text { Preocupación por efectos } \\
\text { futuros de la diabetes }\end{array}$ & 41,3 & 17,3 & 40,1 & 16,6 & 40,4 & 14,9 & 43,8 & 17,0 & 48,2 & 17,9 \\
\hline Calidad de vida (Total DQOL) & 32,7 & 10,3 & 32,3 & 10,1 & 32,9 & 8,9 & 33,6 & 8,9 & 36,4 & 9,7 \\
\hline
\end{tabular}

Tabla 6. Medidas de tendencia central dimensiones por control glicémico y nivel de colesterol examen entre 0 y tres meses atrás

\begin{tabular}{|c|c|c|c|c|c|c|c|c|}
\hline & \multicolumn{2}{|c|}{$\begin{array}{c}\text { Glicemia en } \\
\text { ayunas } \\
90-130 \mathrm{mg} / \mathrm{dl} \\
(21)\end{array}$} & \multicolumn{2}{|c|}{$\begin{array}{l}\text { Glicemia en } \\
\text { ayunas } \\
>131 \mathrm{mg} / \mathrm{dl} \\
(25)\end{array}$} & \multicolumn{2}{|c|}{$\begin{array}{l}\text { Colesterolemia } \\
<\begin{array}{c}200 \mathrm{mg} / \mathrm{dl} \\
\text { (19) }\end{array}\end{array}$} & \multicolumn{2}{|c|}{$\begin{array}{l}\text { Colesterolemia } \\
>\underset{(12)}{201 \mathrm{mg} / \mathrm{dl}}\end{array}$} \\
\hline & $\bar{x}$ & DE & $\bar{x}$ & DE & $\bar{x}$ & DE & $\bar{x}$ & DE \\
\hline Satisfacción con el tratamiento & 37,52 & 13,10 & 39,76 & 11,73 & 36,1 & 11,7 & 42,9 & 10,5 \\
\hline Impacto del tratamiento & 36,24 & 9,47 & 37,84 & 12,15 & 35,3 & 9,3 & 40,2 & 12,6 \\
\hline $\begin{array}{l}\text { Preocupación por aspectos sociales } \\
\text { y vocacionales }\end{array}$ & 15,57 & 16,01 & 13,68 & 7,21 & 16,1 & 16,8 & 15,3 & 6,7 \\
\hline $\begin{array}{l}\text { Preocupación por efectos futuros } \\
\text { de la diabetes }\end{array}$ & 39,52 & 18,50 & 40,60 & 18,44 & 35,3 & 15,5 & 49,6 & 15,4 \\
\hline Calidad de vida (Total DQOL) & 32,21 & 10,80 & 32,97 & 9,10 & 30,7 & 9,9 & 37,0 & 8,1 \\
\hline
\end{tabular}

ciones derivadas de un mal manejo de la enfermedad. El dominio menos afectado fue el que evaluó aspectos sociales y vocacionales, posiblemente por la etapa evolutiva de los encuestados, en su mayoría pacientes mayores de 50 años, en donde los aspectos ligados al desarrollo vocacional y social ya no son tan relevantes en comparación con otros aspectos de su vida.

Las mujeres percibieron una peor CVRS que los hombres en las dimensiones satisfacción con el tratamiento, impacto del tratamiento, preocupación por efectos futuros de la diabetes y además en la CVRS total, hallazgo que coincide con otras investigaciones utilizando similar instrumento ${ }^{15}$. Tal como se ha reportado ${ }^{7}$, el hecho de que el sexo sea significativo en la percepción de la CVRS guarda relación con que las mujeres acuden más a consulta médica, lo cual puede ser un indicador de preocupación sobre su salud o una mayor capacidad de insight sobre dicho estado.

Se encontró que a mayor edad, mejor CVRS percibida en los dominios satisfacción con el tratamiento, preocupación por aspectos sociales y vocacionales, preocupación por efectos futuros de la diabetes y CV total. Posiblemente las personas de edad avanzada podrían presentar un nivel mayor de adaptación y aceptación de la enfermedad. El hecho de que esta relación sólo fuese significativa en las mujeres aporta evidencia al mayor grado de preocupación de éstas por su estado de salud y 
por mantener controlada su enfermedad, lo que ha sido reportado en otras investigaciones ${ }^{11}$.

Los hombres con pareja percibieron mejor su CVRS que los que no la tienen en el dominio satisfacción con el tratamiento. Una posible razón podría ser el factor motivador y reforzador hacia el cumplimiento de la indicación médica por parte de sus parejas, incidiendo en que éstos se adhieran más al tratamiento, provocando por ende mayor satisfacción con los resultados de éste.

Los datos sugieren que a mayor cantidad de personas viviendo con el paciente, peor CVRS percibida en el dominio impacto del tratamiento. Un número mayor de personas viviendo con el paciente aumentaría la posibilidad de que uno o varios de ellos no colaboren en los cambios en el estilo de vida requeridos por el paciente (adaptación a régimen alimenticio, tratamiento farmacológico, inclusión de actividad física diaria), generando que éste perciba mayor dificultad al implementar los cambios en su vida diaria. Este fenómeno se acrecentaría en las mujeres dado que estas percibirían más el cambio producido por los requerimientos del cuidado de la enfermedad, percibiendo menor apoyo social y más carga familiar en relación a la responsabilidad ligada al cuidado de la enfermedad.

En términos generales, mientras más personas que vivan con el paciente lo apoyen en el cuidado de su diabetes, habría una mayor satisfacción con el tratamiento. Sobre esto se ha reportado que pacientes con un alto nivel de satisfacción con el apoyo social percibido, presentan un mayor grado de adaptación a la enfermedad ${ }^{13}$.

Pese a que se ha reportado una menor afectación en la CVRS y una percepción de menor gravedad de su diabetes en pacientes con 5 o menos años desde el diagnóstico ${ }^{16}$, en esta muestra se encontró que a mayor cantidad de años transcurridos desde el diagnóstico, se percibe un menor impacto del tratamiento, posiblemente debido a que a medida que transcurren los años aumentan las posibilidades de presentar complicaciones por la enfermedad, produciendo en el paciente una disminución de su funcionalidad y con esto su CVRS. A su vez, estudios señalan que pacientes con DM2 muestran un proceso gradual en el deterioro de su CV con tendencias relativamente uniformes a medida que avanza la enfermedad en relación con la cronicidad del padecimiento ${ }^{6}$.

Aun cuando se ha reportado que la presencia de comorbilidades asociadas, como la hipertensión, redundaría en una peor $\mathrm{CVRS}^{14}$, no se observa este hecho en la población evaluada. Posiblemente aun cuando los pacientes presentan hipertensión, esta podría estar controlada, por lo que habría que indagar cómo afecta en la CVRS no tan sólo la presencia de una comorbilidad, sino de una comorbilidad no controlada. En este sentido, los pacientes que percibieron complicaciones reportaron una peor CVRS, concordando con lo reportado en otras investigaciones ${ }^{5-7,10}$.

Una limitante que permitiría mejorar esta investigación, sería el acceso a trazadores biológicos ligados al manejo de la enfermedad, como lo son los niveles de glucosa en sangre y hemoglobina glucosilada, pues si bien se tuvo acceso a las fichas de atención médica de los pacientes, estos datos no se encontraban o no estaban actualizados. Lo anterior permitiría conocer la relación entre indicadores objetivos del estado de salud de pacientes con DM2 con su percepción de CVRS, dado que un mejor control glucémico se asocia con una mejor CVRS ${ }^{14}$.

Sería interesante incorporar en futuros estudios otras variables asociadas a la CVRS, tales como, hábitos alimenticios, conductas de autocuidado y adhesión terapéutica, lo cual permitiría desarrollar intervenciones más adecuadas de acuerdo a éstas variables. Dadas las diferencias halladas entre hombres y mujeres, se sugiere además intentar equiparar en futuras investigaciones, la cantidad de participantes por sexo, ya que, como en este caso, una menor cantidad de hombres pudiese constituirse en una limitante a la hora de extrapolar los resultados.

\section{Referencias}

1. Ministerio de Salud. Diabetes mellitus tipo 2. Guía clínica, series guías clínicas MINSAL 2006, 20 (1).

2. Baechler R, Mujica V, Aqueveque X, Ramos L, Soto A. Prevalencia de diabetes mellitus en la VII Región de Chile. Rev Med Chile 2002; 130: 1257-64.

3. López G. Nueva clasificación y criterios diagnósticos de la diabetes mellitus. Rev Med Chile 1998; 126.

4. Alfaro N, Carothers M, González Y. Autopercepción de calidad de vida en adultos mayores con diabetes mellitus tipo 2. Investigación en salud 2006; 8: 152-7.

5. De Pablos P. La calidad de vida se resiente en los pacientes con diabetes. Revista Española de economía de la salud 2008; 7: 79-82. 
6. De los Ríos J, Sánchez J, Barrios P, Guerrero V. Calidad de vida en pacientes con diabetes mellitus tipo 2. Revista Médica IMSS 2004; 42: 109-16.

7. García A, Leiva F, Martos F, García A, Prados D, Sánchez de la Cuesta, et al. Calidad de vida en pacientes con hipertensión arterial y diabetes mellitus tipo 2. Medicina de familia 2001; 2: 29-34.

8. Cardenas V, Pedraza C, Lerma R. Calidad de vida del paciente con diabetes mellitus tipo 2. Ciencia UANL 2005; 8: 351-257.

9. Urzúa A. Calidad de vida relacionada con la salud: Aspectos conceptuales. Rev Med Chile 2010; 138: 341-8.

10. Hervas A, Zabaleta A, De Miguel G, Beldarrain O, Diez. Calidad de vida relacionada con la salud en pacientes con diabetes mellitus tipo 2. Anales Sis San Navarra 2007; 30: 45-52.

11. Urzúa A. Calidad de vida y factores biopsicosociales en patologías médicas crónicas. Terapia Psicológica 2008; 26: 207-15.

12. Arcega A, Lara C, Ponce de León S. Factores relacionados con la percepción subjetiva de la calidad de vida de pacientes con diabetes. Rev Invest Clin 2005; 57: 676-84.

13. Vinaccia S, Fernández H, Quinceno J, López M, Otalvaro C. Calidad de vida relacionada con la salud y apoyo social funcional en pacientes diagnosticados con VIH/ SIDA. Terapia Psicológica 2008; 26: 125-32.

14. Mena F, Martín J, Simal F, Bellido J, Carretero J. Diabetes mellitus tipo 2 y calidad de vida relacionada con la salud: resultados del Estudio Hortega. Anales de Medicina. Interna (Madrid) 2006; 23: 357-60.

15. Robles R, Cortazar J, Sánchez J, Páez F, Nicolini H. Evaluación de la calidad de vida en diabetes mellitus tipo 2: propiedades psicométricas de la versión en español del DQOL. Psicothema 2003; 15: 247-52.

16. López J, Rodríguez R. Adaptación y validación del instrumento de calidad de vida Diabetes 39 en pacientes mexicanos con diabetes mellitus tipo 2. Salud pública de México 2006; 48: 200-11.

17. Jacobson AM, de Groot W, Sanson L. Quality of life Research in patients with Diabetes Mellitus. En: Dimslade J y Baum A. Quality of life in Behavioral Medicine Research. Editorial Lawwrence Erlbaum Associates, Hilldale, NJ. 1995. 